Proceedings

\title{
Modified Commutators Are not Sufficient to Determine a Quantum Gravity Minimal Length Scale
}

\author{
Michael Bishop *, Jaeyeong Lee and Doug Singleton
}

Citation: Lastname, F.; Lastname, F.; Lastname, F. Title. Proceedings 2021, 68, x. https://doi.org/10.3390/xxxxx

Published: date

Publisher's Note: MDPI stays neutral with regard to jurisdictional claims in published maps and institutional affiliations.

Copyright: (c) 2021 by the authors. Submitted for possible open access publication under the terms and conditions of the Creative Commons Attribution (CC BY) license (http://creativecommons.org/licenses /by/4.0/).

\begin{abstract}
In quantum gravity, it is expected that their will be some minimum length scale on possible states of the system. This is derived from modifying the standard quantum position and momentum operators to get a strictly positive lower bound on the uncertainty of position. It is generally thought that a modified commutator of the form $\$[\{\backslash$ hat $x\},\{\backslash$ hat $p\}]=i \backslash$ hbar $\left(1+\backslash\right.$ beta $\left.p^{\wedge} 2\right) \$$ is sufficient to give rise to a minimum length scale. We test this assumption by presenting several different families of modified operators which all lead to the same modified commutator and demonstrating that each family has a different minimum length scale and even no minimal length. We do this by checking the uncertainty in position directly. This is due to the modified operators a subtly different uncertainty principle. The conclusion is that the modification of the operators is the main factor in determining whether there is a minimal length. This fact - that it is the specific form of the modified operators which determine the existence or not of a minimal length scale - can be used to keep or reject specific modifications of the position and momentum operators in theory of quantum gravity. This is joint work with Jaeyeong Lee and Douglas Singleton from the physics department.
\end{abstract}

Keywords: Quantum gravity; minimum length; modified commutators 